\title{
TRANSMUTED BURR TYPE III DISTRIBUTION
}

\section{IBRAHIM B. ABDUL-MONIEM}

Department of Statistics

Higher Institute of Management Sciences in Sohag

Sohag

Egypt

e-mail: ibtaib@hotmail.com

\begin{abstract}
In this paper, we introduce a new distribution called transmuted Burr type III distribution (TBIIID). Some properties of this distribution will be discussed. The estimation of unknown parameters for TIIIBD will be handled using real data.
\end{abstract}

\section{Introduction}

Transmuted distributions can be obtained by adding a real number $\lambda$ $(|\lambda| \leq 1)$ to the cumulative distribution function (CDF), i.e., if we have CDF $G(x)$ of any random variable $X$, then the function

$$
F(x)=(1+\lambda) G(x)-\lambda[G(x)]^{2}, \quad|\lambda| \leq 1,
$$

is called a transmuted distribution, see Shaw and Buckley [14].

2010 Mathematics Subject Classification: 62N05, 90B25.

Keywords and phrases: transmuted distributions, Burr type III distribution, hazard rate function, parameter estimation, moments.

Received July 15, 2015

(ㄷ) 2015 Scientific Advances Publishers 
The probability density function (pdf) corresponding (1) is given by

$$
f(x)=\frac{d F(x)}{d x}=[(1+\lambda)-2 \lambda G(x)] g(x)
$$

where $g(x)$ is the pdf of base distribution.

Many transmuted distributions are proposed. Aryal and Tsokos [4] presented a new generalization of Weibull distribution called the transmuted Weibull distribution. Merovci [11] proposed and studied the various structural properties of the transmuted Rayleigh distribution. Khan and King [9] are introduced the transmuted modified Weibull distribution. Transmuted Lomax distribution is presented by Ashour and Eltehiwy [5]. Elbatal et al. [8] are presented transmuted generalized linear exponential distribution. Merovci and Puka [12] are introduced transmuted Pareto distribution. Transmuted Gompertz distribution is presented by Abdul-Moniem and Seham [1].

A random variable $X$ is said to have Burr type III distribution (BIIID) with two parameters $k$ and $c$ if its pdf is in the form (Burr [7])

$$
g(x)=k c x^{-(c+1)}\left(1+x^{-c}\right)^{-(k+1)} ; \quad x \geq 0, k \text { and } c>0 .
$$

Then the CDF of $g(x)$ in (3) is given by

$$
G(x)=\left(1+x^{-c}\right)^{-k} ; \quad x \geq 0, k \text { and } c>0 .
$$

More details on properties and applications of the BIIID can be found in Burr [7], Lewis [10], AL-Huniti and AL-Dayian [2], and Nayeban et al. [13].

The rest of the paper is organized as follows. In Section 2, we introduce transmuted Burr type III distribution (TBIIID). The traditional moments for TBIIID are given in Section 3. In Section 4, we discussed the parameters estimators using maximum likelihood method. We use a real data set to show that the TBIIID can be a better model than one based on the BIIID in Section 5. Section 6 concludes the paper. 


\section{Transmuted Burr Type III Distribution}

Using (1) and (4), we can define the CDF of transmuted Burr type III distribution (TBIIID) as follows:

$$
F(x)=\left(1+x^{-c}\right)^{-k}\left[1+\lambda-\lambda\left(1+x^{-c}\right)^{-k}\right] ; x \geq 0, c, k>0 \text {, and }|\lambda| \leq 1 \text {. }
$$

The pdf of TBIIID is

$$
\begin{aligned}
f(x)=k c x^{-(c+1)}\left(1+x^{-c}\right)^{-(k+1)} & {\left[1+\lambda-2 \lambda\left(1+x^{-c}\right)^{-k}\right] ; } \\
x & \geq 0, c, k>0 \text {, and }|\lambda| \leq 1 .
\end{aligned}
$$

Figures 1 and 2, depict the behaviour of pdf and CDF for some parameter values.

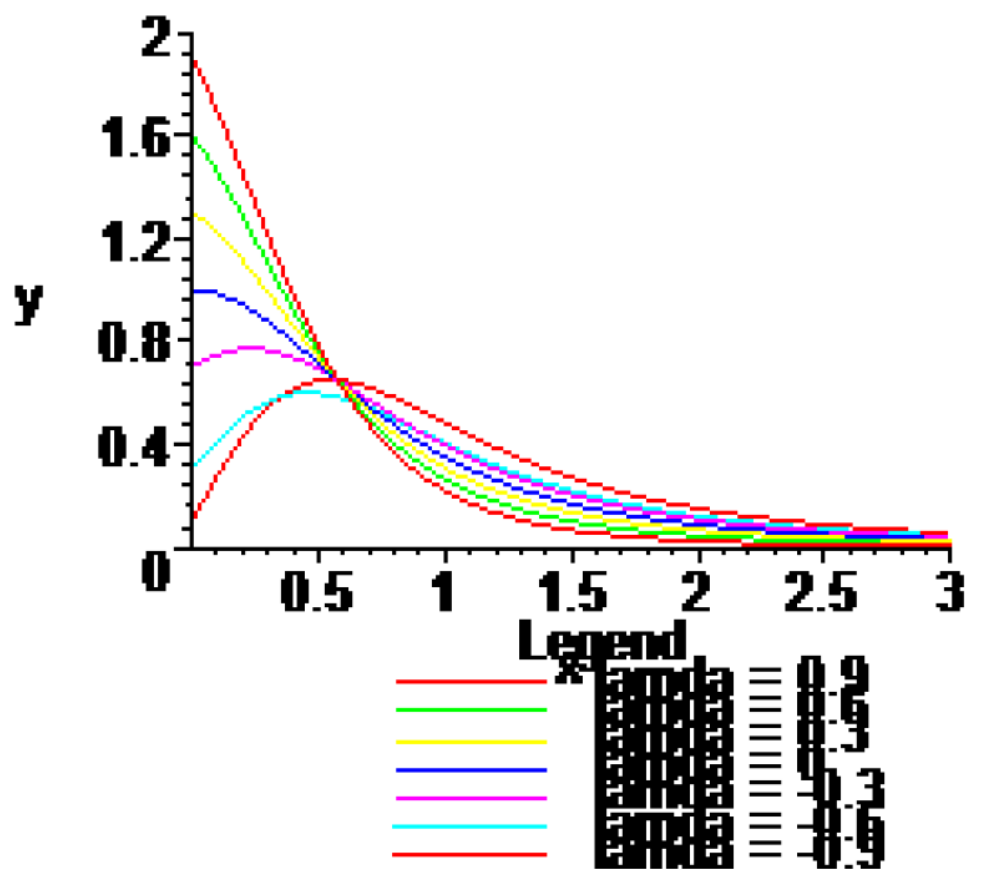

Figure 1. Plot of pdf at $c=2, k=0.5$ with different values of $\lambda$. 


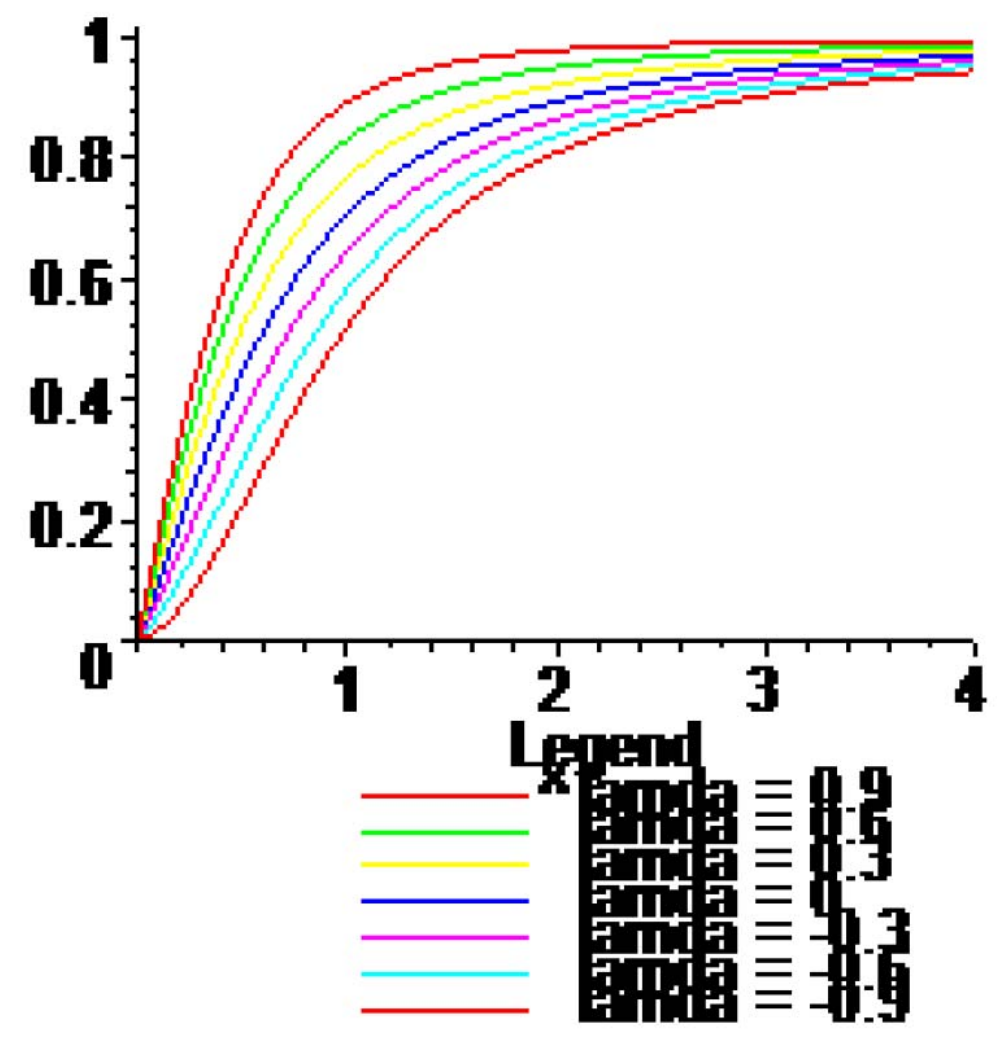

Figure 2. Plot of CDF at $c=2, k=0.5$ with different values of $\lambda$.

The reliability function $R(x)$, the hazard rate function (HRF) $h_{\lambda}(x)$, and the reversed hazard rate function (RHRF) $h_{\lambda}^{*}(x)$ for TBIIID are in the following forms:

$$
\begin{aligned}
& R(x)=1-\left(1+x^{-c}\right)^{-k}\left[1+\lambda-\lambda\left(1+x^{-c}\right)^{-k}\right] ; x \geq 0, c, k>0, \text { and }|\lambda| \leq 1 \\
& h_{\lambda}(x)=\frac{k c x^{-(c+1)}\left[1+\lambda-2 \lambda\left(1+x^{-c}\right)^{-k}\right]}{\left(1+x^{-c}\right)^{(k+1)}-\left(1+x^{-c}\right)\left[1+\lambda-\lambda\left(1+x^{-c}\right)^{-k}\right]} ; \\
& x \geq 0, c, k>0 \text { and }|\lambda| \leq 1,(8)
\end{aligned}
$$


and

$$
h_{\lambda}^{*}(x)=h^{*}(x)-\frac{\lambda h^{*}(x)}{\left[(1+\lambda)\left(1+x^{-c}\right)^{k}-\lambda\right]} ; \quad x \geq 0, c, k>0 \text {, and }|\lambda| \leq 1,(9)
$$

where $h(x)$ and $h^{*}(x)$ are the HRF and RHRF for the Burr type III distribution, respectively. Figure 3 depict the behaviour of HRF for some parameter values.

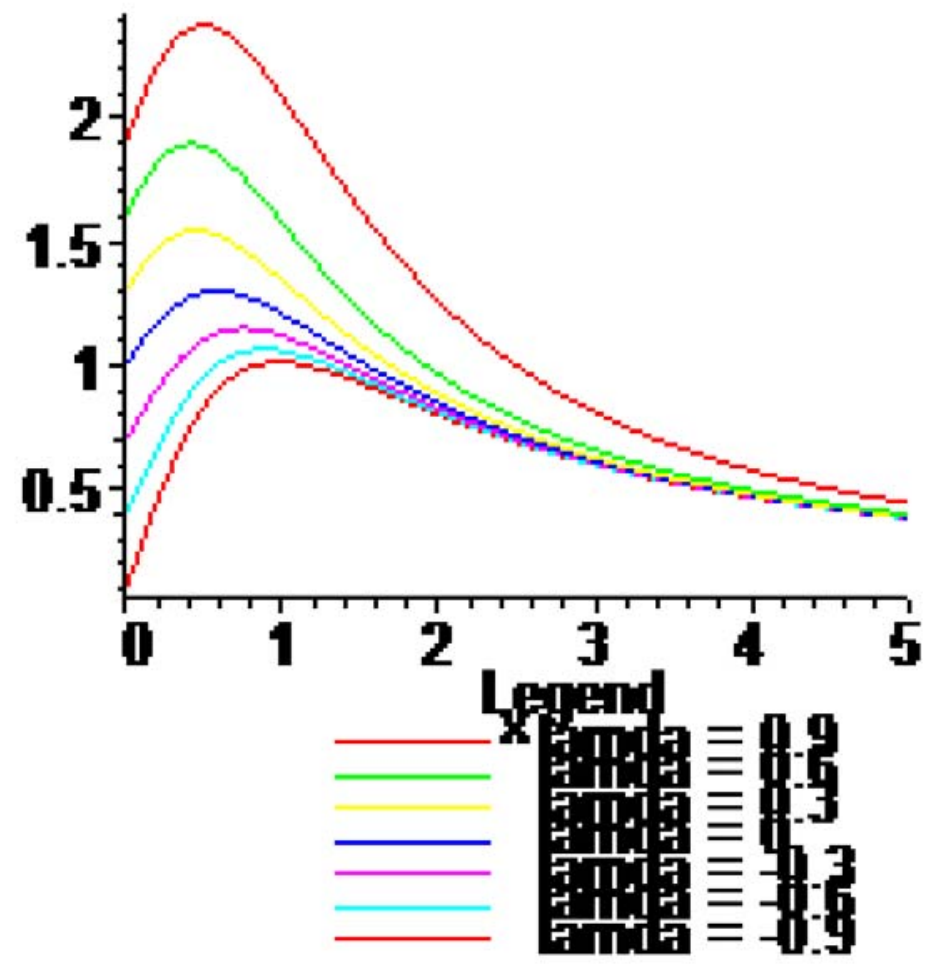

Figure 3. Plot of HRF at $c=2, k=0.5$ with different values of $\lambda$.

The median of TBIIID is

$$
x_{0.5}=\left\{\left[\frac{\sqrt{1+\lambda^{2}}+1+\lambda}{2 \lambda}\right]^{\frac{-1}{k}}-1\right\}
$$




\section{Traditional Moments for TBIIID}

The $r$-th traditional moments for TBIIID is

$$
\mu_{r}^{\prime}=E\left(X^{r}\right)=k c \int_{0}^{\infty} x^{r-(c+1)}\left(1+x^{-c}\right)^{-(k+1)}\left[1+\lambda-2 \lambda\left(1+x^{-c}\right)^{-k}\right] d x
$$

Using the transformation $y=\left(1+x^{-c}\right)^{-1}$, we get

$$
\begin{aligned}
\mu_{r}^{\prime} & =k \int_{0}^{1}\left(\frac{1-y}{y}\right)^{-\frac{r}{c}} y^{k-1}\left[1+\lambda-2 \lambda y^{k}\right] d y \\
& =k\left[(1+\lambda) \beta\left(1-\frac{r}{c}, k+\frac{r}{c}\right)-2 \lambda \beta\left(1-\frac{r}{c}, 2 k+\frac{r}{c}\right)\right], r=1,2, \ldots
\end{aligned}
$$

The first two moments can be obtained by taking $r=1$ and 2 in (10) as follows:

$$
\mu_{1}^{\prime}=k\left[(1+\lambda) \beta\left(1-\frac{1}{c}, k+\frac{1}{c}\right)-2 \lambda \beta\left(1-\frac{1}{c}, 2 k+\frac{1}{c}\right)\right],
$$

and

$$
\mu_{2}^{\prime}=k\left[(1+\lambda) \beta\left(1-\frac{2}{c}, k+\frac{2}{c}\right)-2 \lambda \beta\left(1-\frac{2}{c}, 2 k+\frac{2}{c}\right)\right] .
$$

The variance and coefficient of variation (CV) for TBIIID are

$$
\begin{aligned}
\operatorname{Var}(X)= & k\left[(1+\lambda) \beta\left(1-\frac{2}{c}, k+\frac{2}{c}\right)-2 \lambda \beta\left(1-\frac{2}{c}, 2 k+\frac{2}{c}\right)\right] \\
& -k^{2}\left[(1+\lambda) \beta\left(1-\frac{1}{c}, k+\frac{1}{c}\right)-2 \lambda \beta\left(1-\frac{1}{c}, 2 k+\frac{1}{c}\right)\right]^{2},
\end{aligned}
$$

and

$$
C V=\sqrt{\frac{\left[(1+\lambda) \beta\left(1-\frac{2}{c}, k+\frac{2}{c}\right)-2 \lambda \beta\left(1-\frac{2}{c}, 2 k+\frac{2}{c}\right)\right]}{k\left[(1+\lambda) \beta\left(1-\frac{1}{c}, k+\frac{1}{c}\right)-2 \lambda \beta\left(1-\frac{1}{c}, 2 k+\frac{1}{c}\right)\right]^{2}}-1}
$$




\section{Parameters Estimators}

In this section, we consider maximum likelihood estimators (MLE) of TBIIID. Let $x_{1}, x_{2}, \ldots, x_{n}$ be a random sample of size $n$ from TBIIID, then the log-likelihood function $L L$ can be written as

$$
\begin{array}{r}
L L \propto n[\ln (k)+\ln (c)]-(c+1) \sum_{i=1}^{n} \ln \left(x_{i}\right)-(k+1) \sum_{i=1}^{n} \ln \left(1+\left(x_{i}\right)^{-c}\right) \\
+\sum_{i=1}^{n} \ln \left[1+\lambda-2 \lambda\left(1+\left(x_{i}\right)^{-c}\right)^{-k}\right] .
\end{array}
$$

The normal equations become

$$
\begin{gathered}
\frac{\partial L L}{\partial c}=\frac{n}{c}-\sum_{i=1}^{n} \ln \left(x_{i}\right)+(k+1) \sum_{i=1}^{n} \frac{\left(x_{i}\right)^{-c} \ln \left(x_{i}\right)}{1+x^{-c}} \\
-2 \lambda k \sum_{i=1}^{n} \frac{\left(x_{i}\right)^{-c} \ln \left(x_{i}\right)\left(1+\left(x_{i}\right)^{-c}\right)^{-(k+1)}}{1+\lambda-2 \lambda\left(1+\left(x_{i}\right)^{-c}\right)^{-k}}, \\
\frac{\partial L L}{\partial k}=\frac{n}{k}-\sum_{i=1}^{n} \ln \left(1+x^{-c}\right)+2 \lambda \sum_{i=1}^{n} \frac{\left(1+x^{-c}\right)^{-k} \ln \left(1+x^{-c}\right)}{1+\lambda-2 \lambda\left(1+x^{-c}\right)^{-k}} \\
\frac{\partial L L}{\partial \lambda}=\sum_{i=1}^{n} \frac{1-2\left(1+\left(x_{i}\right)^{-c}\right)^{-k}}{1+\lambda-2 \lambda\left(1+\left(x_{i}\right)^{-c}\right)^{-k}} .
\end{gathered}
$$

The MLE of $c, k$, and $\lambda$ can be obtain by solving the Equations (15), (16), and (17) using $\frac{\partial L L}{\partial c}=0, \frac{\partial L L}{\partial k}=0$, and $\frac{\partial L L}{\partial \lambda}=0$.

\section{Application of TBIIID}

In this section, we use a real data set to show that the TBIIID can be a better model than one based on the BIIID. We consider a data set of the 
life of fatigue fracture of Kevlar 373/epoxy that are subject to constant pressure at the $90 \%$ stress level until all had failed, so we have complete data with the exact times of failure. For previous studies with the data sets, see Andrews and Herzberg [3], Barlow et al. [6], and Abdul-Moniem and Seham [1]. These data are:

0.0251, 0.0886, 0.0891, 0.2501, 0.3113, 0.3451, 0.4763, 0.5650, 0.5671, 0.6566, 0.6748, $0.6751,0.6753,0.7696,0.8375,0.8391,0.8425,0.8645,0.8851,0.9113,0.9120,0.9836$, 1.0483, 1.0596, 1.0773, 1.1733, 1.2570, 1.2766, 1.2985, 1.3211, 1.3503, 1.3551, 1.4595, $1.4880,1.5728,1.5733,1.7083,1.7263,1.7460,1.7630,1.7746,1.8275,1.8375,1.8503$, 1.8808, 1.8878, 1.8881, 1.9316, 1.9558, 2.0048, 2.0408, 2.0903, 2.1093, 2.1330, 2.2100, 2.2460, 2.2878, 2.3203, 2.3470, 2.3513, 2.4951, 2.5260, 2.9911, 3.0256, 3.2678, 3.4045, 3.4846, 3.7433, 3.7455, 3.9143, 4.8073, 5.4005, 5.4435, 5.5295, 6.5541, 9.0960.

The LR statistics to test the hypotheses $\mathrm{H}_{0}: \lambda=0$ versus $\mathrm{H}_{1}: \lambda \neq 0$ : $\omega=6.74>6.635=\chi_{1}^{2}(\alpha=0.01)$, so we reject the null hypotheses.

In order to compare the distributions, we consider some other criterion like K-S (Kolmogorow-Smirnov), -2LL, AIC (Akaike Information Criterion), AICC (Akaike Information Criterion Corrected), and BIC (Bayesian Information Criterion) for the real data set. The best distribution corresponds to lower K-S, -2LL, AIC, AICC, and BIC values: $\mathrm{KS}=\max _{1 \leq i \leq n}\left(F\left(x_{i}\right)-\frac{i-1}{n}, \frac{1}{n}-F\left(x_{i}\right)\right), \mathrm{AIC}=2 m-2 L L, \mathrm{AICC}=\mathrm{AIC}+$ $\frac{2 m(m+1)}{n-m-1}$, and $\mathrm{BIC}=m \log (n)-2 L L$, where $m$ is the number of parameters in the statistical model, $n$ the sample size, and $L L$ is the maximized value of the log likelihood function for the estimated model. Also, here for calculating the values of K-S, we use the sample estimates of $k, c$, and $\lambda$. Table 1 shows parameter MLEs to each one of the two fitted distributions. 
Table 1. Estimated parameters of the TBIIID and BIIID

\begin{tabular}{|c|c|c|}
\hline Model & Parameters estimates & $-L L$ \\
\hline TBIIID & $\hat{k}=0.97711, \hat{c}=1.85661, \hat{\lambda}=-0.71802$ & 125.045 \\
\hline BIIID & $\hat{k}=1.45899, \hat{c}=1.69913$ & 128.415 \\
\hline
\end{tabular}

Table 2 shows the values of K-S, -2LL, AIC, AICC, and BIC values.

Table 2. Criteria for comparison

\begin{tabular}{|c|c|c|c|c|c|}
\hline Model & K-S & $-2 \mathrm{LL}$ & AIC & AICC & BIC \\
\hline TBIIID & 0.135 & 250.089 & 256.089 & 257.589 & 263.082 \\
\hline BIIID & 0.150 & 256.830 & 260.830 & 261.536 & 265.491 \\
\hline
\end{tabular}

The values in Table 2 indicate that the TBIIID leads to a better fit than BIIID. We can see the same notes in the next figure.

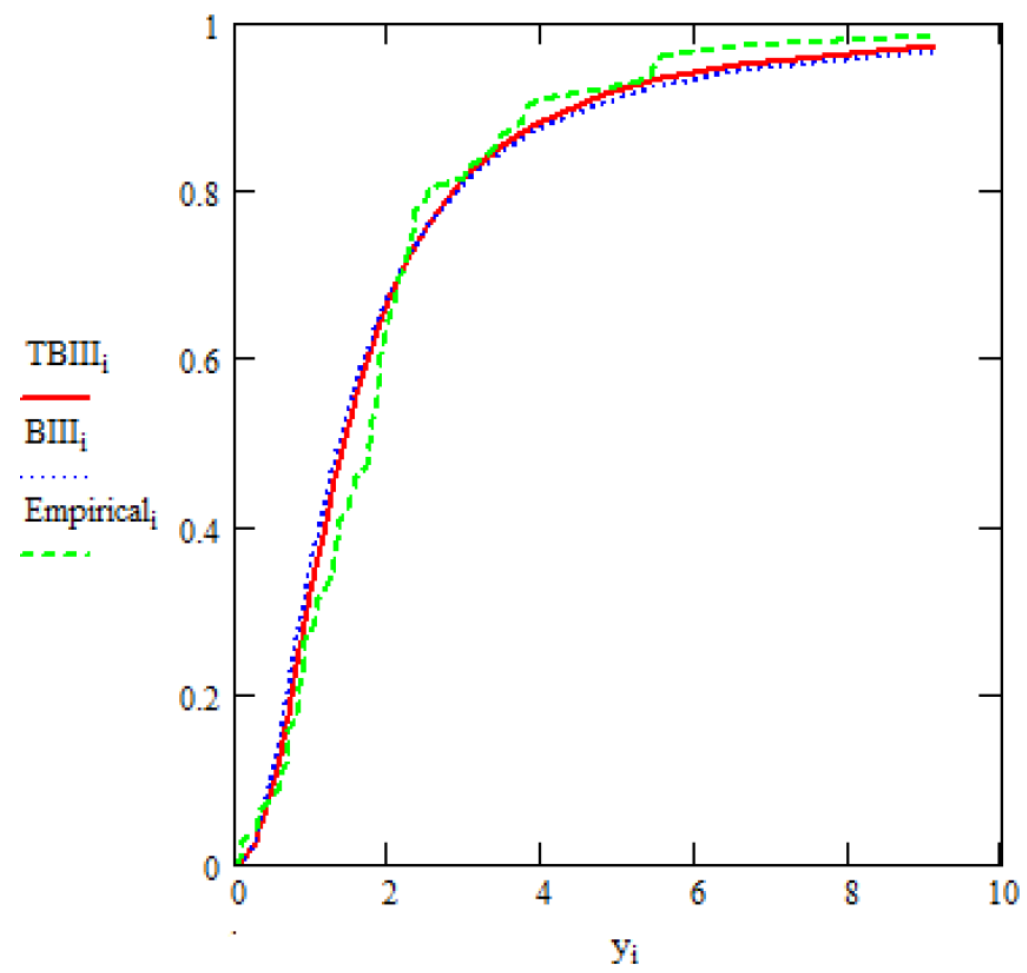

Figure 4. Empirical, fitted TBIIID and BIIID CDF of the above data. 


\section{Conclusion}

In this article, we introduce a new generalization of the Burr type III distribution called transmuted Burr type III distribution and presented its theoretical properties. The estimation of parameters is approached by the method of maximum likelihood. We consider the likelihood ratio statistic to compare the model with its baseline model. An application of the transmuted Burr type III distribution to real data show that the new distribution can be used quite effectively to provide better than the Burr type III distribution.

\section{References}

[1] I. B. Abdul-Moniem and M. Seham, Transmuted Gompertz distribution, Computational and Applied Mathematics 1(3) (2015), 88-96.

[2] A. A. AL-Huniti and G. R. AL-Dayian, Discrete Burr type III distribution, American Journal of Mathematics and Statistics 2(5) (2012), 145-152.

[3] D. F. Andrews and A. M. Herzberg, Data: A Collection of Problems from Many Fields for the Student and Research Worker, Springer Series in Statistics, New York, 1985.

[4] G. R. Aryal and Ch. P. Tsokos, Transmuted Weibull distribution: A generalization of the Weibull probability distribution, Europian Journal of Pure and Applied Mathematics 4(2) (2011), 89-102.

[5] S. K. Ashour and M. A. Eltehiwy, Transmuted Lomax distribution, American Journal of Applied Mathematics and Statistics 1(6) (2013), 121-127.

[6] R. E. Barlow, R. H. Toland and T. Freeman, A Bayesian Analysis of Stress Rupture Life of Kevlar 49/epoxy Sphere-cal Pressure Vessels, in 'Proc. Conference on Applications of Statistics', Marcel Dekker, New York, 1984.

[7] I. W. Burr, Cumulative frequency functions, Annals of Mathematical Statistics 13 (1942), 215-232.

[8] I. Elbatal, L. S. Diab and N. A. Abdul-Alim, Transmuted generalized linear exponential distribution, International Journal of Computer Applications 83(17) (2013), 29-37.

[9] M. S. Khan and R. King, Transmuted modified Weibull distribution: A generalization of the modified Weibull probability distribution, European Journal of Pure and Applied Mathematics 6 (2013), 66-88.

[10] A. W. Lewis, The Burr Distribution as a General Parametric Family in Survivorship and Reliability Theory Applications, Ph.D. Thesis, Department of Biostatistics, University of North Carolina, Chapel Hill, 1981. 
[11] F. Merovci, Transmuted Rayleigh distribution, Austrian Journal of Statistics 42(1) (2013), 21-31.

[12] F. Merovci and L. Puka, Transmuted Pareto distribution, ProbStat Forum 7 (2014), $1-11$.

[13] S. Nayeban, A. H. Rezaei Roknabadi and G. R. Mohtashami Borzadaran, Comparative study of Bhattacharyya and Kshirsagar bounds in Burr XII and Burr III distributions, Chilean Journal of Statistics 5(1) (2014), 103-118.

[14] W. T. Shaw and I. R. Buckley, The alchemy of probability distributions: Beyond Gram-Charlier expansions, and a skew-kurtotic-normal distribution from a rank transmutation map, arXiv preprint arXiv: 0901.0434, (2009). 